\title{
A method to improve the determination of wave perturbations close to the tropopause by using a digital filter
}

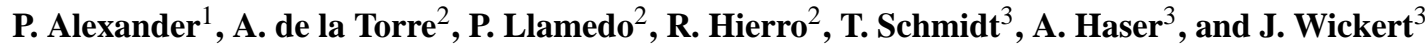 \\ ${ }^{1}$ Departamento de Física, Facultad de Ciencias Exactas y Naturales, Universidad de Buenos Aires, \\ 1428 Buenos Aires, Argentina \\ ${ }^{2}$ Facultad de Ingeniería, Universidad Austral, Av. J. de Garay 125, 1063 Buenos Aires, Argentina \\ ${ }^{3}$ Helmholtz Centre Potsdam, GFZ German Research Centre for Geosciences, Telegrafenberg A17, \\ Potsdam, 14473, Germany
}

Received: 23 December 2010 - Published in Atmos. Meas. Tech. Discuss.: 23 February 2011

Revised: 11 August 2011 - Accepted: 26 August 2011 - Published: 8 September 2011

\begin{abstract}
GPS radio occultation satellite data allowed to analyze in the last decade for the first time a large amount of atmospheric temperature profiles including both the troposphere and the stratosphere all over the globe. Wave amplitude enhancements have been systematically observed around tropopause levels, which are apparently due to artifacts generated by any digital filter used to isolate the wave components from these data. We present a new filtering method which can be equally applied to temperature or refractivity profiles. It was tested with synthetic temperature data based on NCEP reanalyes and observed wave climatologies and it was also statistically validated with GPS radio occultation profiles from the COSMIC mission. The suggested technique significantly reduces artificial enhancements around the tropopause, mainly at low latitudes, where a sharp lapse rate change usually exists. This represents an improvement in comparison to previous applications of standard filters. In addition it would allow the study of longer vertical wavelengths than previously done with other filtering procedures.
\end{abstract}

\section{Introduction}

Atmospheric waves play an important role in the transport of momentum and energy in the lower and middle atmosphere and have a key protagonism in the general circulation. In the last decades, a variety of techniques have provided an ever increasing amount of data to describe these dynamical aspects: radar, lidar, aircraft, rocket, radiosonde and satellites (e.g. Tsuda et al., 1991; Nastrom and Fritts, 1992; Eckermann and Vincent, 1993; Wu et al., 2006).

Among the satellite methods we will briefly describe one of them due to its importance in the present work. A Global Positioning System (GPS) radio occultation (RO) occurs whenever a transmitter on board a satellite from the GPS network at an altitude about $20000 \mathrm{~km}$ rises or sets from the standpoint of a low Earth orbit satellite receiver at a height of some $100 \mathrm{~km}$ and the ray traverses the atmospheric limb. The aim of the GPS RO is to detect the perturbation in Doppler frequency produced through refraction of the signal by the Earth's atmosphere in the limb path between the transmitter and the receiver. This information can be converted into vertical atmospheric refractivity, pressure, density and temperature profiles. The advantages as compared to other methods are that this technique is nearly an instantaneous snapshot (typically $1 \mathrm{~min}$ as compared to the much longer atmospheric processes), it has a global coverage, sub-Kelvin accuracy in temperature measurements from the upper troposphere to the lower stratosphere, good vertical resolution, and it is not interrupted by clouds or bad weather conditions.

Measured variables in the atmosphere may be often separated into background and perturbation structures. The former is assumed to possess much larger time or space scales and is not affected by the fluctuations. The latter is considered to have zero mean over the longer scales. Different methods may be chosen to separate the original data into both parts and their characteristics and assumptions may lead to different results (Zülicke and Peters, 2006). 


\section{Separation of background and perturbations}

When going from the troposphere to the stratosphere some medium properties may undergo significant changes. As a consequence, some theoretical work has considered partial reflections in the tropopause. However, in soundings no definite indication of this phenomenon has yet been found. There has been remarkably little investigation of this issue in recent years and we are only aware of one work which might have detected a possible partial reflection in the tropopause (Smith et al., 2008). Regardless of this lack of certainty on this important and complex issue, perturbations around the tropopause have been estimated in recent wave climatologies (e.g. Alexander et al., 2008).

When vertical profiles of temperature $T$ data from whatever observational technique are processed for wave activity analysis, singular problems emerge for the separation process between background $T_{\mathrm{B}}$ and perturbation $T^{\prime}$ components. One alternative to break off background and fluctuations is to use a polynomial function to fit the former (e.g. Allen and Vincent, 1995; Vincent et al., 1997). Guest et al. (2000) discussed how to choose the most adequate polynomial order. However, this technique allows no direct control of the wavelength range to be isolated. Another option is to use a digital filter. The larger wavelengths are separated because they are assumed to represent the background, whereas the smallest ones are eliminated because they are considered to be waves in a spectral region outside the study's scope of interest or just instrumental or atmospheric noise. The remaining perturbations are those of interest to the analysis. Bandpass filters often damp the wave amplitudes and polynomial fits of the background fields sometimes create overshoots (Zülicke and Peters, 2006).

Moreover, the sharp lapse rate change around the tropopause, particularly at low latitudes, strongly affects the performance of any separation method. The background cannot be adequately reproduced, mainly leading to an artificial enhancement of the amplitudes of the waves that are present in the fluctuating structure to be studied. This has been concluded after almost a decade of work on global wave climatologies obtained from GPS RO satellite data (see, e.g. Steiner and Kirchengast, 2000; Tsuda et al., 2000; Ratnam et al., 2004; de la Torre et al., 2006; Namboothiri et al., 2008), which allowed to analyze, for the first time, a large amount of temperature profiles including both the troposphere and stratosphere. A large difference close to the LRT (lapse rate tropopause) between the temperature profile measured by a GPS RO example and the corresponding background determined by a filter (Scavuzzo et al., 1998) may be seen in Fig. 1. This kind of problem has initially led to over-estimations of amplitudes close to the tropopause and once acknowledged has precluded the possibility of performing studies encompassing the troposphere and stratosphere within the same data-set. A first partial solution was to restrict any data analysis to each atmospheric layer or to only

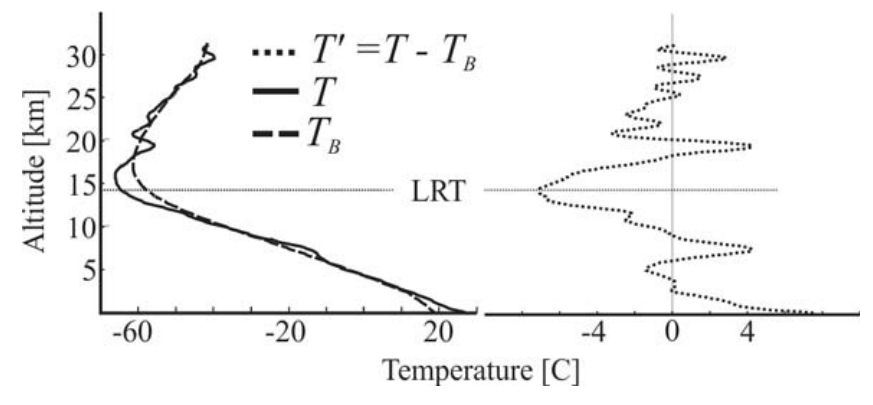

Fig. 1. A GPS RO temperature profile (solid), the background temperature determined by a filter (dashed) and their difference (dotted) on the right (Satellite CHAMP, lon $=293.81^{\circ}$, lat $=-18.55^{\circ}$, 30 May 2001, 01:42 UTC).

one of them. At the time, the possible inference of vertical wavelengths larger than around $15 \mathrm{~km}$ was not possible in this kind of works. In addition, wave activity in the tropopause region could not be realistically determined.

Schmidt et al. (2008) discussed the global gravity wave activity derived from CHAllenging Minisatellite Payload (CHAMP) GPS RO data by separating tropospheric and stratospheric data and applying a filter in each region. The total temperature background profile was constructed from the two parts and compared to the filtering applied to the complete altitude range. The separate filtering method significantly reduced the usually observed artificial wave activity enhancement in the tropopause region. An assessment of the significant errors introduced by the tropopause artificial enhancement when using a digital filter for the "complete" and "separate" methods applied to GPS RO temperature profiles has been recently carried out by de la Torre et al. (2010). We will compare below these two alternatives with the double filtering method.

Another possibility for separating background and waves emerged recently when the Constellation Observing System for Meteorology, Ionosphere, and Climate (COSMIC) GPS RO data became available. Its spatial and temporal occurrence density is much larger than in previous missions, which may be sufficient for the determination of a background temperature by averaging at each height over as small as possible (according to minimum sampling size criterium) latitude/longitude bins and time intervals (see, e.g. Alexander et al., 2008; Wang and Alexander, 2010). These authors constructed for each grid cell of given longitude and latitude bands a background temperature profile over a defined time interval. The oscillation components were obtained by subtracting individual temperature profiles from the appropriate background. However, for older GPS RO missions or for other observational techniques the number of profiles per cell is too scarce to generate representative backgrounds, and the method is no longer meaningful. In addition, some researchers may legitimately consider that temporal or spatial resolutions higher than the ones used by the above authors 
are needed to adequately separate both parts. All these cases require a solution to the tropopause problem.

\section{A new filtering method}

If filters with ideal cut-offs would exist, part of the problem would still be there because the tropopause kink usually departs from a sinusoid or any other function that may be used as a basis. In addition, real filters do not yield ideal spectral components isolations (side effects as amplitude reduction for example) and may need some manual fine tuning procedures in optimizing their performance. After a "perfect" band-pass is used there should be no remaining components at wavelengths outside of the considered range. However, real filters behave differently, so using them more than once in a given way could a priori improve the removal of undesired wavelengths. We tried different alternatives and empirically found a method that we call double filtering, which basically implies the use of the same filter twice. No additional processing procedures like removal of trends or prewhitening were used. We checked the method with two different filters, because it could a priori lead to non-coincident outcomes. An explanation of each of both filters may be respectively found in Scavuzzo et al. (1998) and Schönwiese (2006). Both are non-recursive and include a Kaiser window (Kaiser, 1966) in the height domain to minimize filtering artifacts due to the non-infinite extension of the data. The two filters led to almost identical results, so we show below results only for the one described by Scavuzzo et al. (1998). The filter response to a unit impulse located in the middle within 400 data points may be seen in Fig. 2. The equations that define the filter coefficients are rather cumbersome. Figure 2 shows in a much simpler way the distribution of the coefficients (we recall that they are by definition the response to a unit pulse in every non-recursive filter). The sum of all coefficients is 1 . For larger or smaller cutoffs $\lambda$ (in our case 50) the shape of the response would we wider or narrower. If the cut-off of the filter was ideal, then a double filtering would always lead to null modifications by the second processing, as the longest wavelengths would already have been eliminated in the first step.

We applied the method to temperature and refractivity. In the neutral dry atmosphere refractivity is proportional to total pressure and inversely proportional to temperature. Refractivity has the advantages that it is a lower level product of RO (see, e.g. Kursinski et al., 1997) and it typically has no abrupt behavior around the tropopause. However, the drawback is that it has an exponential decrease, so any inappropriate representation of the background may probably lead to an artificial amplification of any fluctuation.

The new method has two steps: (i) use the bandpass filtering to isolate the wavelength range of interest (separate the background and eliminate the noise), (ii) perform on the isolated perturbation component a low pass filtering with a
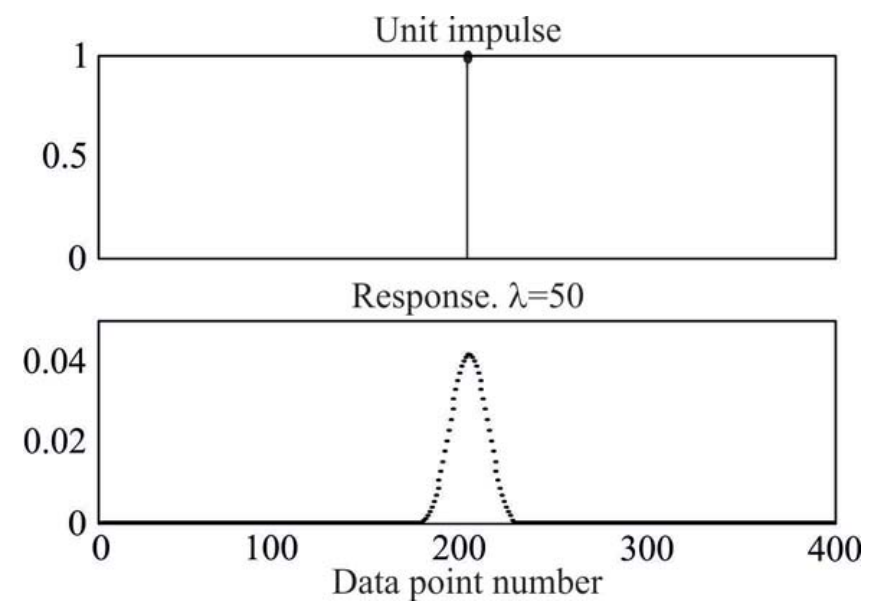

Fig. 2. A unit pulse located at data point number 200 and the corresponding filter response with a low pass cut off equal to 50 times the uniform interval size on the horizontal axis between neighboring data points.

cutoff that is larger than or equal to the bandpass upper limit (remove large wavelengths representing background behavior or trends still present and force a zero mean). Keep in mind that the tropopause kink in temperature can be viewed as the surrounding of a long sinusoidal peak. In all the cases studied below we applied in the first step a bandpass between 1 and $10 \mathrm{~km}$ and in the second step a cutoff of $10 \mathrm{~km}$.

\section{Results and discussion}

We show the results of some examples in Figs. 3 and 4 for double filtered temperature $T_{\mathrm{D}}^{\prime}$. Biases and non zero means may be seen around the tropopause for a standard filtering procedure. These problems are attenuated after applying the suggested second step to find any trend or bias in the isolated perturbation component (AUX). Amplitudes, wavelengths and phase variabilities are not always retained. We repeated the procedure with refractivity and met with similar success. In Figs. 5 and 6 we show the technique applied to relative (ratio of perturbation and total components) temperature and refractivity in two examples. Note that the nearly exponential decrease of refractivity with increasing height cannot be appropriately followed by the bandpass filter well above the tropopause and fluctuations become magnified and biased at this stage. However, the problem is corrected by the second step in the double filtering method (temperature and refractivity oscillations show nearly equal relative amplitudes and opposite signs as expected in gravity waves).

We also show now how the new method may improve the estimation of wave activity in altitude intervals including the tropopause, which is the zone affected by the filtering artifact. Wave activity is usually quantified by potential energy, which is nearly equivalent to the average relative 
a)

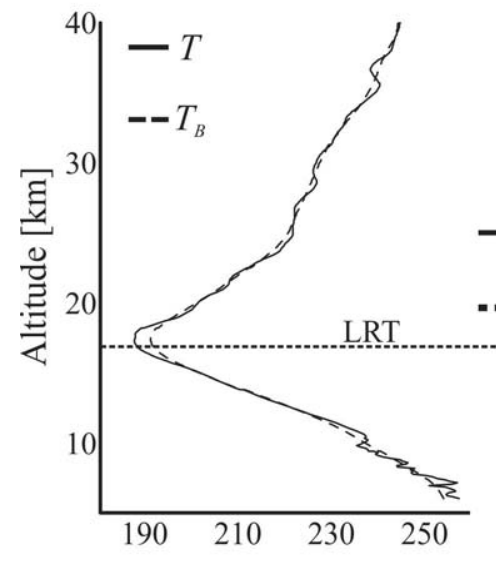

b)

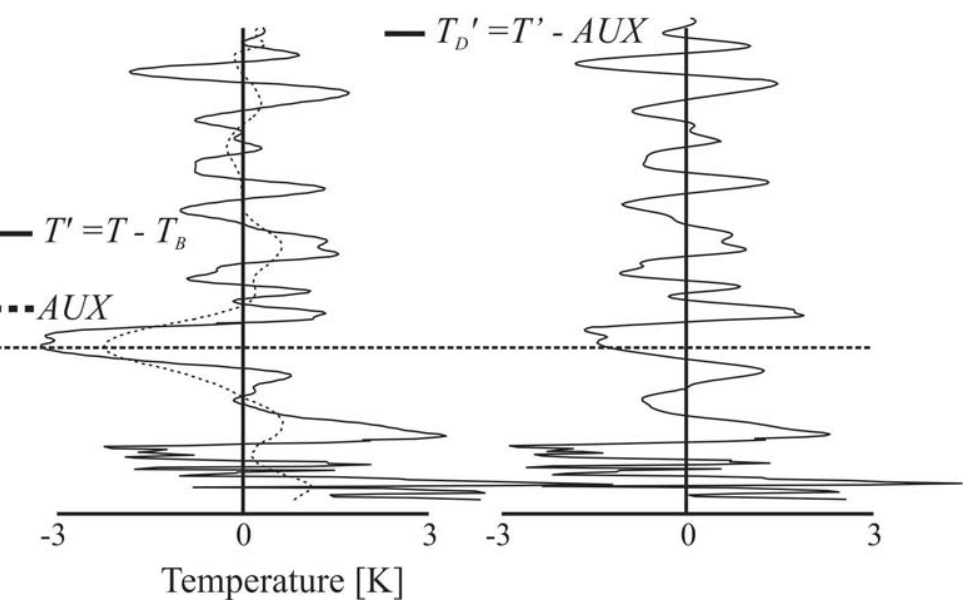

Fig. 3. Shown is (a) the RO temperature profile (solid) and the determined background temperature (dashed), (b) the bandpass filtered temperature (solid) and the background corresponding to large wavelengths (dashed), and (c) the double filtered temperature (Satellite COSMIC 1 , lon $=239.36^{\circ}$, lat $=-2.38^{\circ}, 29$ June $2010,00: 23$ UTC).

a)

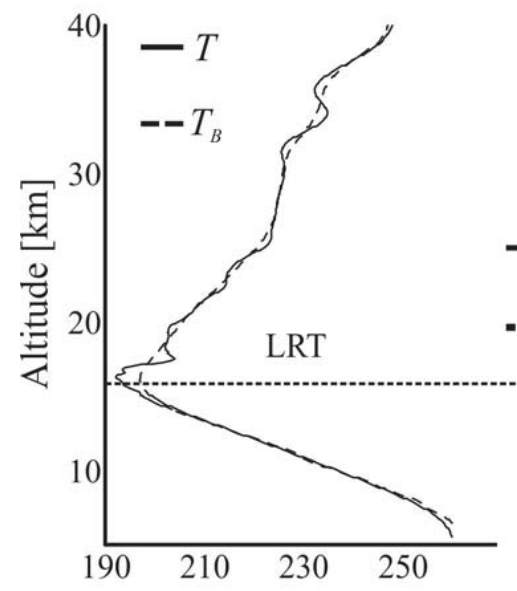

b)

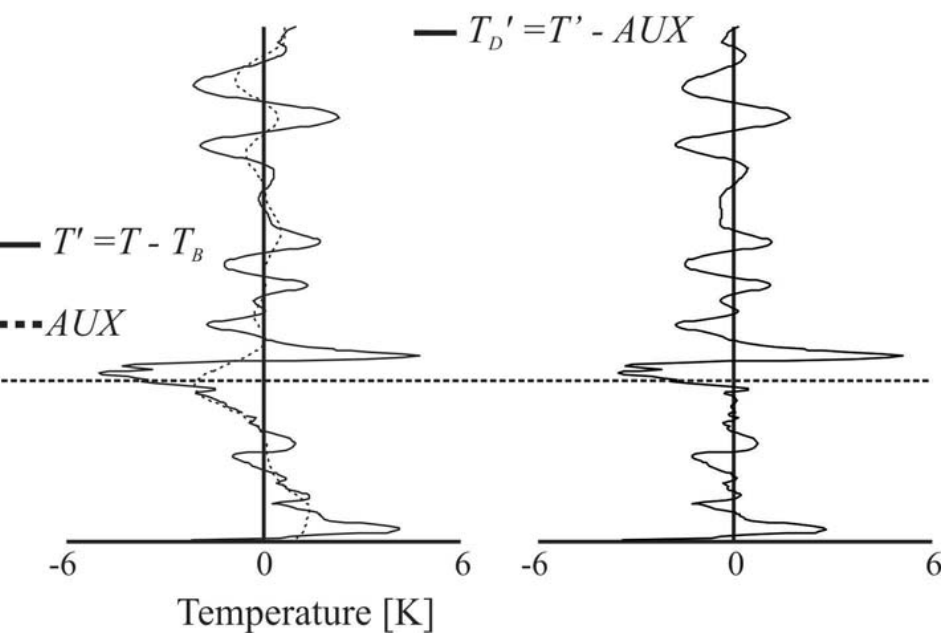

Fig. 4. Same as Fig. 3 for a second GPS RO event (Satellite COSMIC 1, lon $=44.47^{\circ}$, lat $=7.01^{\circ}, 21$ March 2010, 16:20 UTC).

temperature $T^{\prime} / T$ variance (Tsuda et al., 2000). We will show below the latter quantity, which is the square of the relative temperature averaged over a given height interval (relative temperature profiles have been shown in the examples in Figs. 5 and 6). We first created synthetic temperature data between 4 and $27 \mathrm{~km}$ height by adding two known components, a background $T_{\mathrm{B}}$ plus a perturbation $T^{\prime}$. The background temperature was selected from National Center for Environmental Prediction (NCEP) reanalyses data at intervals of $5^{\circ}$ latitude, zonally averaged and randomly chosen between January 1997 and December 2007. The largest altitude interval available for all latitudes and times is $4-27 \mathrm{~km}$. The perturbation temperature was generated as a superposition of monochromatic waves with vertical wavelengths that ranged between 1 and $15 \mathrm{~km}$ every $0.5 \mathrm{~km}$ and random amplitudes and phases. A modulation function finally matches the perturbations to observed climatologies. More details of the buildup of the synthetic profiles is given by de la Torre et al. (2010). For each latitude there were 500 created profiles, which allowed to calculate mean (reference) values of average temperature variance (mean average variance over 500 profiles) and their uncertainty (the standard deviation of the average variance over 500 profiles). This in turn was compared with the calculation of the same quantities after applying the "complete", "separate" and double filtering methods described above. The results are shown in Fig. 7. Notice that as defined above the relative variance gives a nondimensional indirect indication of $T^{\prime}$ (complete method) and 


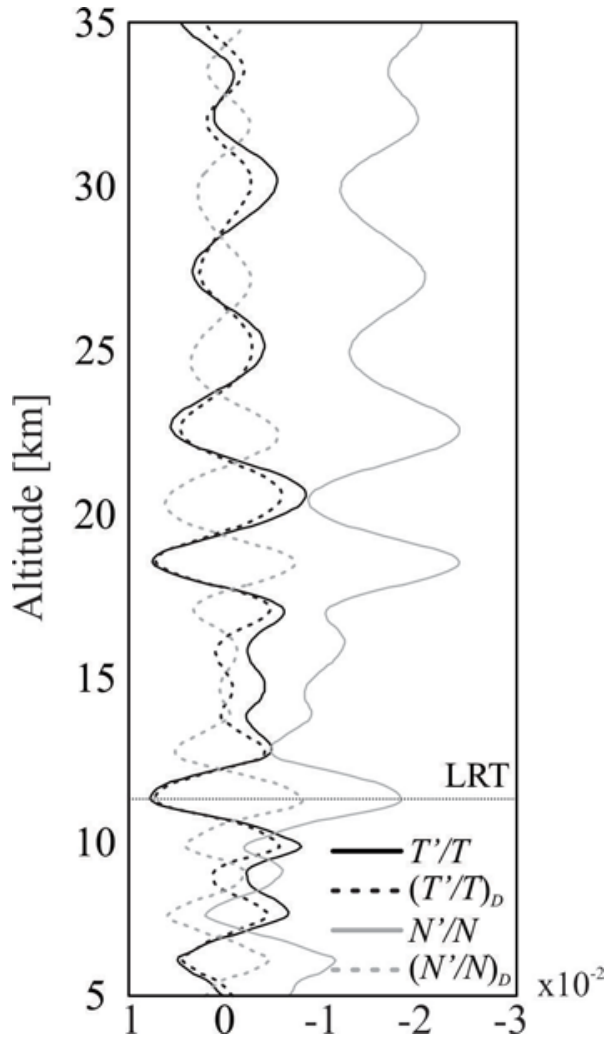

Fig. 5. Bandpass (solid) and double filtered (dashed) relative temperature (black) and refractivity (gray) profiles of a GPS RO event (Satellite COSMIC 1 , lon $=18.06^{\circ}$, lat $=-36.30^{\circ}$, 19 July 2010, 19:07 UTC).

$T_{\mathrm{D}}^{\prime}$ (double filtering method), so both corresponding profiles are also rough quantitative representations of the average temperature perturbation for each method. It may be clearly seen that the option that best follows the "true" values is the double filtering method. The other two methods exhibit order of magnitude differences at some latitudes. Near the equator the application of the complete method leads typically to 6 fold variances as compared to the "true" reference values (roughly 3 against 0.5 ), whereas the double filtering leads to differences around $20 \%$ (0.6 against 0.5$)$. Somewhat lower but similar discrepancies may be found at middle and high latitudes (the typical relative variance values change). The energy of the long scales that was not removed by the first filtering but by the second is represented essentially by the difference between the complete and the double filtering profiles at each latitude. The largest discrepancies occur at low latitudes due to the sharp lapse rate change at the tropopause. Typical kink variation with latitude can be seen in Fig. 8, where we show mean temperature profiles from zonally averaged NCEP reanalyses for January 2007 (Southern and Northern Hemisphere summer and winter, respectively). It is noteworthy that in a small area (mainly close to latitude $-20^{\circ}$ ) the double filtering method underestimates the "true" wave activity in Fig. 7. This strange effect hap-

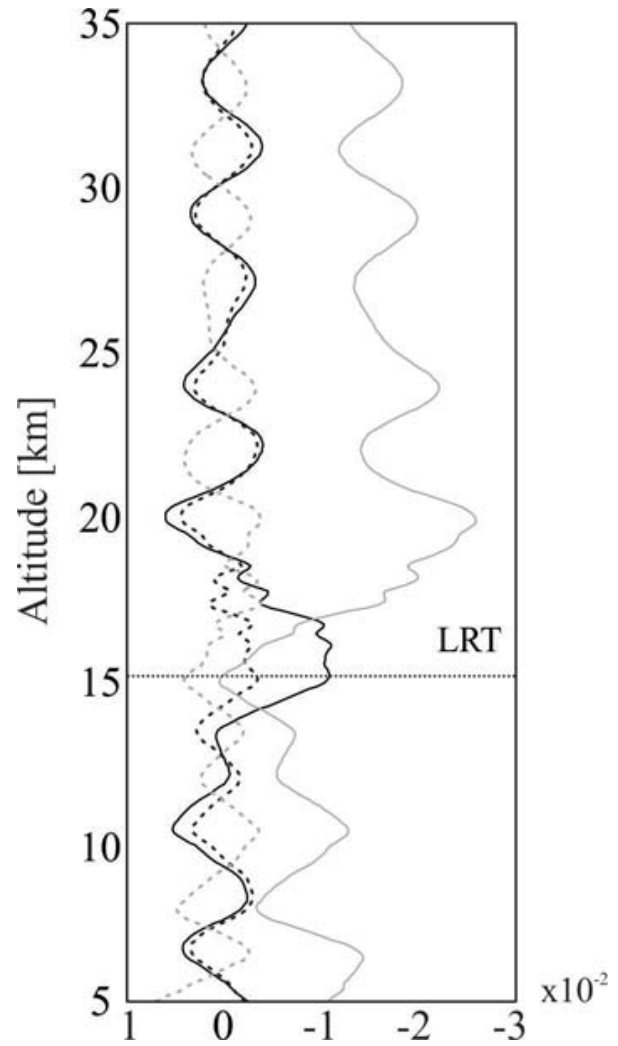

Fig. 6. Same as Fig. 5 for a second GPS RO event (Satellite COSMIC 1 , lon $=95.29^{\circ}$, lat $=-23.02^{\circ}$, 19 July 2010, 00:38 UTC).

pens because an excessive detrending occurs for some particular temperature profiles. We finally recall that the separate method (Schmidt et al., 2008) breaks off the measured temperature profile in two parts at the cold point tropopause (CPT). Each of both backgrounds is found separately. They are then joined together to find the perturbations from the troposphere to the stratosphere. The two parts often do not match nicely at the CTP. A consequence of this problem is that wave amplitudes become somewhat overestimated (not as much as in the complete method) and therefore also the energy values become magnified.

A statistics on reduction of average potential energy of the double filtering method with respect to the complete method per geographical cell $\left(5 \times 5^{\circ}\right)$ based on one month of global COSMIC GPS RO observational data is shown in Fig. 9. In agreement with the above simulations, significantly larger values may be observed mainly at low latitudes for the complete method, which may be expected to stem from the difficulties in adequately separating the tropopause kink according to the above results. We also included a panel where the background was removed according to the method developed by Alexander et al. (2008), which we call "grid cell background procedure". There are global similarities between this alternative and the double filtering method, but the 


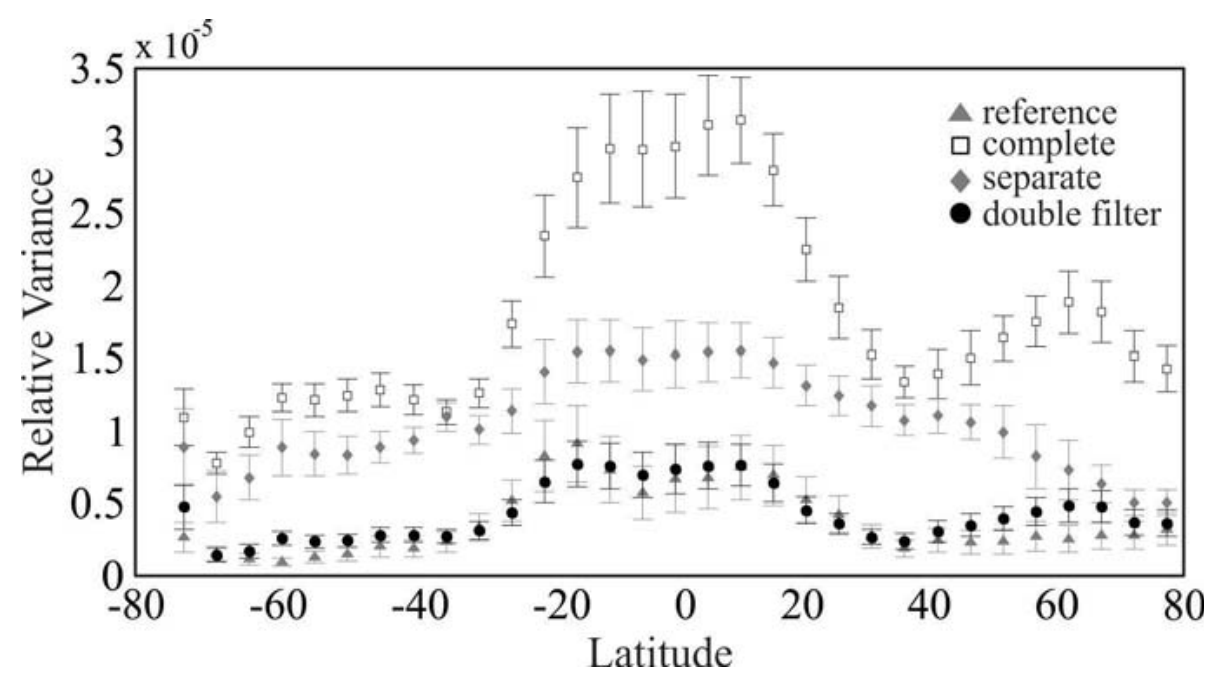

Fig. 7. Simulated mean average relative temperature variance and its uncertainty in the height interval $4-27 \mathrm{~km}$ against latitude: reference calculation (triangles) and the results for the complete (squares), separate (rhomboids) and double filtering (circles) procedures.

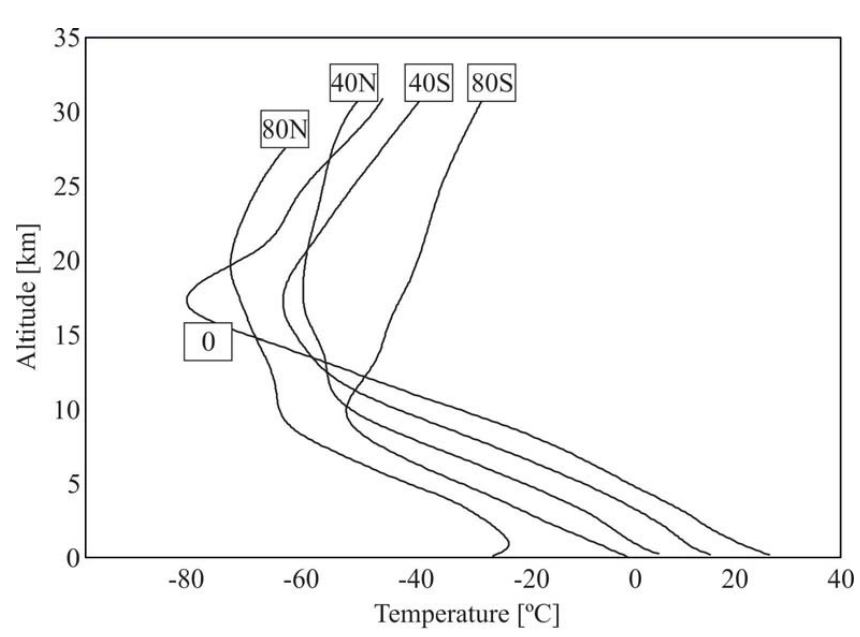

Fig. 8. Low, middle and high latitude mean temperature profiles from NCEP reanalyses zonally averaged during January 2007.

former exhibits some noticeable activity close to the North Pole, which is even more remarkable near to the South Pole. This is an artifact that stems from the need by this method to have a sufficient number of cases to produce statistically significant mean background temperature for the same cell size as in the other two methods. We made calculations for the grid cell background procedure with 1 month means. When zooming in the data we found that the temperature profiles close to the poles exhibited a significant variability during June 2008. We could have chosen the option followed by the developers of the method, who used shorter time averages for the background temperature (1 week), but then the size of the cells would have been different than in the other two methods shown in the figure. This fact reveals the del-

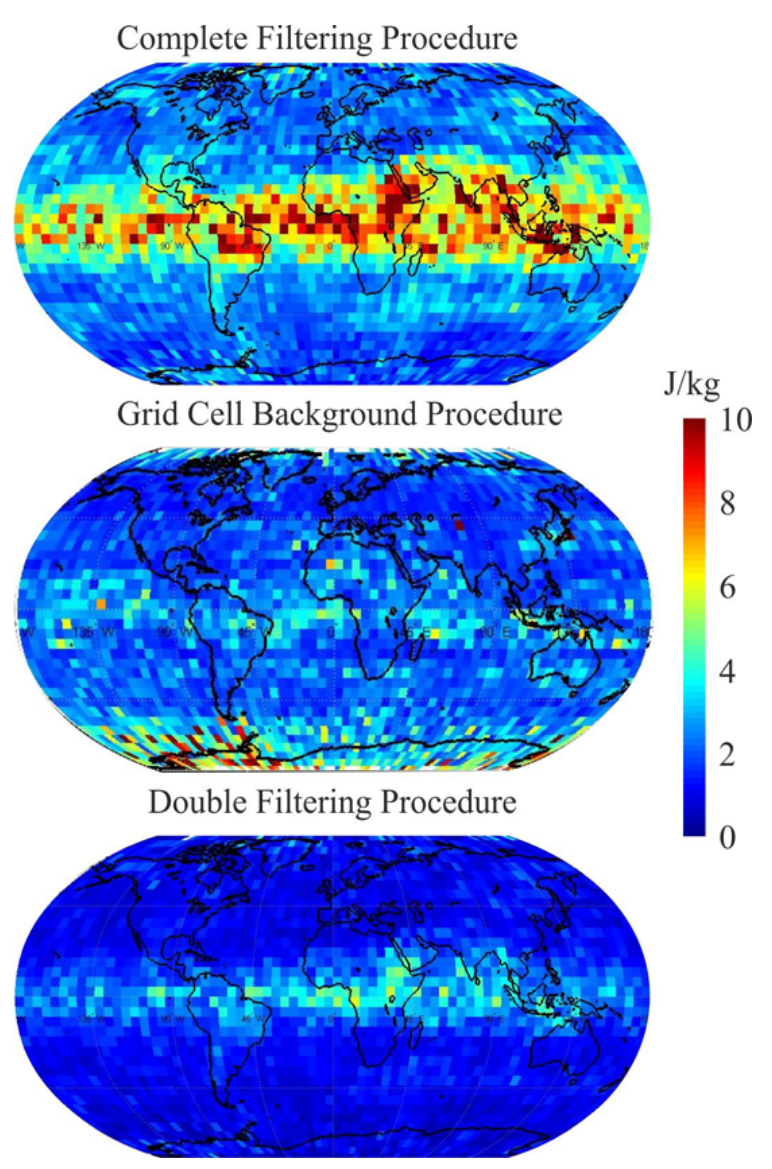

Fig. 9. Average potential energy per $5 \times 5^{\circ}$ cells between $5 \mathrm{~km}$ and $35 \mathrm{~km}$ height by the double filtering method, the grid cell background procedure and the complete method, based on global COSMIC GPS RO data from June 2008. 
icate compromise that is needed in the grid cell background procedure between time and space intervals. Alexander et al. (2008) already noticed that the potential energy values may artificially increase as the considered time intervals become longer. In addition, we should mention that they included altitude intervals from the tropopause to the stratosphere in some of their calculations, but they did not incorporate the troposphere.

In brief, we were able to satisfactorily circumvent the tropopause problem with the possibility of using either temperature or refractivity, as preferred by the user. This improvement would allow the calculation of gravity wave activity, now including longer vertical wavelengths than previously considered, as ultrafast Kelvin waves around the equator (e.g. Canziani et al., 1994).

\section{Conclusions}

The artificial enhancement of gravity wave induced perturbations that is produced by the use of standard filtering procedures around the tropopause is significantly reduced with the suggested double filtering method. Two examples representing difficult cases are shown, which are low latitude temperature profiles with a sharp change in lapse rate. From simulations it may be concluded that the new technique also allows to obtain better estimations of wave activity around the tropopause. The improvement implied by the suggested method will allow the inclusion of waves with longer vertical wavelengths in future calculations along the troposphere and stratosphere. In addition, amplitudes calculated from temperature and refractivity radio occultation data were shown in two examples to produce results which have no significant differences.

Acknowledgements. Manuscript prepared under grants UBA X004, CONICET PIP 11220090100649 and ANPCYT PICT 1999. P. Alexander and A. de la Torre are members of CONICET and P. Llamedo and R. Hierro are respectively holders of a fellowship of CONICET and ANPCYT. We thank CHAMP and COSMIC GPS RO data downloaded from COSMIC homepage.

Edited by: A. K. Steiner

\section{References}

Alexander, P. A., Tsuda, T., Kawatani, Y., and Takahashi, M.: Global distribution of atmospheric waves in the equatorial upper troposphere and lower stratosphere: COSMIC observations of wave mean flow interactions, J. Geophys. Res., 113, D24115, doi:10.1029/2008JD010039, 2008.

Allen, S. J. and Vincent, R. A.: Gravity wave activity in the lower atmosphere: Seasonal and latitudinal variations, J. Geophys. Res., 100, 1327-1350, 1995.

Canziani, P. O., Holton, J. R., Fishbein, E., Froidevaux, L., and Waters, J. W.: Equatorial Kelvin waves: A UARS MLS view, J. Atmos. Sci., 51, 3053-3076, 1994. de la Torre, A., Schmidt, T., and Wickert, J.: A global analysis of wave potential energy in the lower stratosphere derived from 5 years of GPS radio occultation data with CHAMP, Geophys. Res. Lett., 33, L24809, doi:10.1029/2006GL027696, 2006.

de la Torre, A., Llamedo, P., Alexander, P., Schmidt, T., and Wickert, J.: Estimated errors in a global gravity wave climatology from GPS radio occultation temperature profiles, Adv. Space Res., 46, 174-179, 2010.

Eckermann, S. D. and Vincent, R. A.: VHF radar observations of gravity-wave production by cold fronts over southern Australia, J. Atmos. Sci., 50, 785-806, 1993.

Guest, F. M., Reeder, M. J., Marks, C. J., and Karoly, D. J.: Inertiagravity waves observed in the lower stratosphere over Macquarie Island, J. Atmos. Sci., 57, 737-752, 2000.

Kaiser, J. F.: Digital Filters, in: System Analysis by Digital Computer, edited by: Kuo, F. F. and Kaiser, J. F., Wiley, New York, 1966.

Kursinski, E. R., Hajj, G. A., Schofield, J. T., Linfield, R. P., and Hardy, K. R.: Observing Earth's atmosphere with radio occultation measurements using the Global Positioning System, J. Geophys. Res., 102, 23429-23465, 1997.

Namboothiri, S. P., Jiang, J. H., Kishore, P., Igarashi, K., Ao, C. O., and Romans, L. J.: CHAMP observations of global gravity wave fields in the troposphere and stratosphere, J. Geophys. Res., 113, D07102, doi:10.1029/2007JD008912, 2008.

Nastrom, G. D. and Fritts, D. C.: Sources of mesoscale variability of gravity waves, I, topographic excitation, J. Atmos. Sci., 49, 101-110, 1992.

Ratnam, M. V., Tetzlaff, G., and Jacobi, C.: Global and seasonal variations of stratospheric gravity wave activity deduced from the CHAMP/GPS satellite, J. Atmos. Sci., 61, 1610-1620, 2004.

Scavuzzo, C. M., Lamfri, M. A., Teitelbaum, H., and Lott, F.: A study of the low-frequency inertio-gravity waves observed during the Pyrénés Experiment, J. Geophys. Res., 103, 1747-1758, 1998.

Schmidt, T., de la Torre, A., and Wickert, J.: Global gravity wave activity in the tropopause region from CHAMP radio occultation data, Geophys. Res. Lett., 35, L16807, doi:10.1029/2008GL034986, 2008.

Schönwiese, C. D.: Praktische Statistik für Meteorologen und Geowissenschaftler, 4th edn., Borntraeger Gebrueder, Berlin, 2006.

Smith, R. B., Woods, B. K., Jensen, J., Cooper, W. A., Doyle, J. D., Jiang, Q., and Grubisic, V.: Mountain waves entering the stratosphere, J. Atmos. Sci., 65, 2543-2562, doi:10.1175/2007JAS2598.1, 2008.

Steiner, A. K. and Kirchengast, G.: Gravity wave spectra from GPS/MET occultation observations, J. Atmos. Ocean. Tech., 17, 495-503, 2000.

Tsuda, T., VanZandt, T. E., Mizumoto, M., Kato, S., and Fukao, S.: Spectral analysis of temperature and BruntVäisälä frequency fluctuations observed by radiosondes, J. Geophys. Res., 96, 17265-17278, 1991.

Tsuda, T., Nishida, M., Rocken, C., and Ware, R. H.: A global morphology of gravity wave activity in the stratosphere revealed by the GPS occultation data (GPS/MET), J. Geophys. Res., 105, 7257-7273, 2000.

Vincent, R. A., Allen, S. J., and Eckermann, S. D.: Gravity-wave parameters in the lower stratosphere, NATO ASI Series I, Global Environmental Change, vol. 50, Springer-Verlag, 7-25, 1997. 
Wang, L. and Alexander, M. J.: Global estimates of gravity wave parameters from GPS radio occultation temperature data, J. Geophys. Res., 115, D21122, doi:10.1029/2010JD013860, 2010.

Wu, D. L., Preusse, P., Eckermann, S. D., Jiang, J. H., de la Torre Juárez, M., Coy, L., and Wang, D. Y.: Remote sounding of atmospheric gravity waves with satellite limb and nadir techniques, Adv. Space Res., 37, 2269-2277, doi:10.1016/j.asr.2005.07.031, 2006.
Zülicke, C. and Peters, D.: Simulation of inertia-gravity waves in a poleward-breaking Rossby wave, J. Atmos. Sci., 63, 3253-3276, 2006. 